\title{
Diseño de un modelo de innovación para incremento de la competitividad en la producción de nochebuena (Euphorbia Pulcherrima) bajo invernadero
}

\section{Design of an innovation model to increase competitiveness in the production of poinsettia (Euphorbia Pulcherrima) under greenhouse}

\author{
MÉNDEZ-HERNÁNDEZ, José Luis †**, ROMERO-CRUZ, Clara, MENDIETA-REYES, Jesús Fidel y \\ GUTIÉRREZ-LÓPEZ, Laura
}

Instituto Tecnológico Superior de San Martín Texmelucan

ID $1^{\text {er }}$ Autor: José Luis, Méndez-Hernández / ORC ID: 0000-002-89461051, Researcher ID Thomson: S-7848-2018, arXiv Author ID: joseluis\#1, CVU CONACYT ID: 367084

ID $1^{\text {er }}$ Coautor: Clara, Romero-Cruz / ORC ID: 0000-0002-69395712, Researcher ID Thomson: S-5947-2018, arXiv Author ID: ClaraRomeroCruz, CVU CONACYT ID: 947137

ID $2^{\text {do }}$ Coautor: Jesus Fidel, Mendieta-Reyes / ORC ID: 0000-0002-1454-6675, arXiv Author ID: jesus.fidel, CVU CONACYT ID: 999282

ID $3^{\text {er }}$ Coautor: Laura, Gutierrez-Lopez / ORC ID: 0000-0002-03934664, arXiv Author ID: LauraGtz, CVU CONACYT ID: IT19F163

\begin{abstract}
Design a theoretical model of innovation focused on the agribusiness sector A review of the scientific literature on the topic of agribusiness innovation was carried out in the main scientific platforms stories such as Thomson Reuters Web of Science, etc., the following variables were detected; business cooperation, technological capacity, knowledge creation, government programs, agribusiness groups and productive diversification, a questionnaire was applied to the producers of Euphorbia Pulcherrima, the data affected are as follows; business cooperation, government programs, knowledge creation, technological capacity and agribusiness clusters exposed to a correlation coefficient (Spearman) of 0.625 to 0.805 , however the variable productive diversification shows low correlation level of 0.313 to 0.327 . The original contribution of this proposal is based on the statistical support of the variables and the unification of variables through a theoretical model.
\end{abstract}

Model, Innovación, Competitive

\begin{abstract}
Resumen
Diseñar un modelo teórico de innovación enfocado al sector agroindustrial Se realizó una revisión de la literatura científica sobre el tema de innovación agroindustrial en las principales plataformas científicas tales como, Web of Science de Thomson Reuters, etc., se detectaron las siguientes variables; cooperación empresarial, capacidad tecnológica, creación de conocimiento, programas gubernamentales, agrupamientos agroindustriales y diversificación productiva, se aplicó un cuestionario a los productores de Euphorbia Pulcherrima, los datos obtenidos son los siguientes ; cooperación empresarial, programas gubernamentales, creación del conocimiento, capacidad tecnológica y agrupamientos agroindustriales muestran un coeficiente de correlación (Spearman) de 0.625 a 0.805 , sin embargo la variable diversificación productiva muestra bajo nivel de correlación de 0.313 a 0.327. La contribución original de la presente propuesta se basa en el soporte estadísticos de las variables y unificación de variables mediante un modelo teórico.
\end{abstract}

Citación: MÉNDEZ-HERNÁNDEZ, José Luis, ROMERO-CRUZ, Clara, MENDIETA-REYES, Jesús Fidel y GUTIÉRREZ-LÓPEZ, Laura. Diseño de un modelo de innovación para incremento de la competitividad en la producción de nochebuena (Euphorbia Pulcherrima) bajo invernadero. Revista del Diseño Innovativo. 2019. 3-8: 24-31

\footnotetext{
* Correspondencia del Autor (Correo electrónico: joseluis.mendez@ upaep.edu.mx)

$\dagger$ Investigador contribuyendo como primer autor.
} 


\section{Introducción}

Las empresas requieren capacidades para adquirir y aplicar conocimientos innovadores para crear nuevos productos y servicios, prácticas de comercialización y la apertura de nuevos mercados, tecnología y organización de la producción, formas de organización y gerencia empresarial. También, para crear nuevas formas de relacionarse en redes empresariales y cadenas de valor y con proveedores de servicios de desarrollo empresarial y financiamiento, universidades y otros potenciales de conocimientos innovadores (Cummings, 2013). El contexto actual en el que se encuentra la innovación tecnológica es de constante cambio. La generación, el acceso y la adaptación de conocimientos, aunados a la aparición y difusión acelerada de nuevas tecnologías, exigen adaptaciones permanentes, lo que sin duda constituye en un reto para la sociedad en su conjunto. El crecimiento económico y social, el mantenimiento del empleo y la competitividad, pasan inevitablemente por la innovación y la transferencia tecnológica. (Zarazúa et al., 2009).

\section{Fundamentación Teórica}

En la literatura revisada se analizaron diferentes propuestas relacionados a la incorporación de modelos de innovación específicamente en el sector agroindustrial donde la base es el financiamiento de los proyectos relacionados con el modelo y viceversa, para ello se identificaron 3 modelos que particularmente se adaptan a la relación que guarda el producto con la tecnología y el comportamiento del agricultor.

\section{Modelo de adaptación}

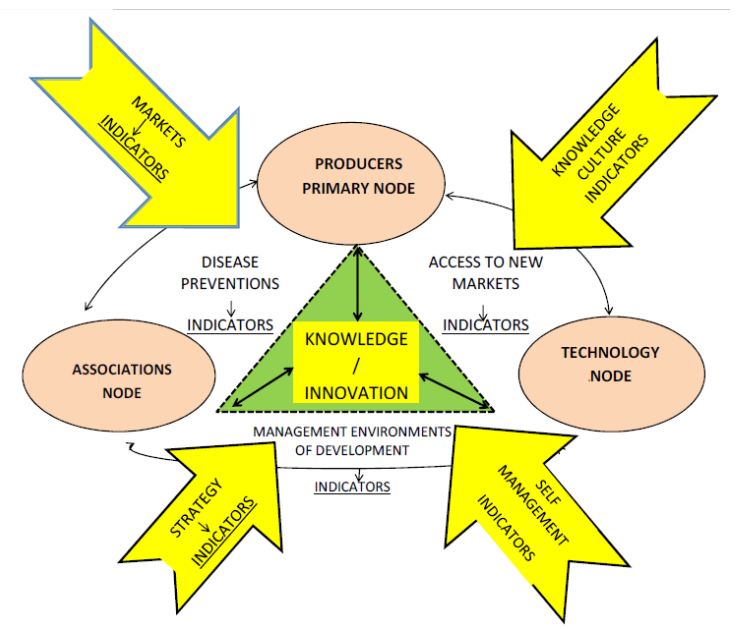

Figura 1 Knowledge Management Model for FruitHorticultural Agroindustry Case: Córdoba - Colombia
En este esquema se está presentando como el eje central, producto de esta interacción de los mencionados linfáticos, el "conocimiento" y la "innovación", que se traduce como la razón de tal esquematización y el beneficio de esto tiene repercusiones en los niveles de desarrollo de la agroindustria (Pérez y García, 2013). La propuesta del modelo está basada en: conectividad, colaboración, economía basada en el conocimiento, tecnología de redes, la oportunidad de aprender unos de otros y tener un conocimiento cada vez más especializado y productivo.

\section{Modelo de adaptación}

La innovación por parte de las empresas portuguesas tiene un efecto positivo en su mercado y rendimiento financiero y viceversa, y que existe retroalimentación positiva entre el de entrada, de salida y de rendimiento fases de los procesos de innovación realizadas por ellos. Comprender la relación precisa que une la innovación y el desempeño de las empresas no es sólo es importante para el liderazgo de las empresas individuales, sino también para la planificación y políticas a nivel nacional.

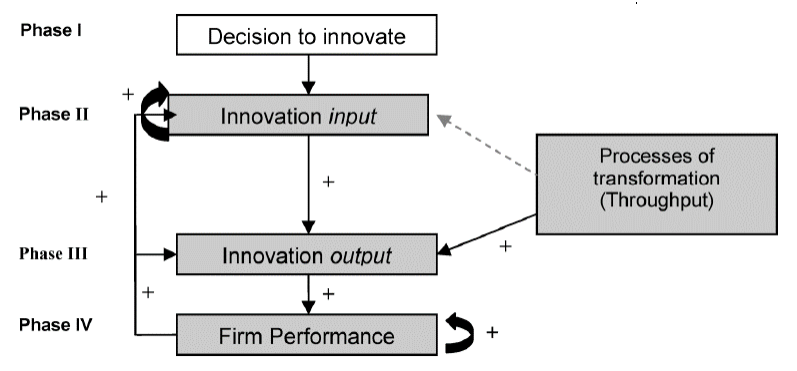

Figura 2 Innovation and the performance of Portuguese businesses, Marques Gerry and Covelo, 2011

Los resultados de esta investigación sugiere que la competencia "schumpeteriana" está todavía muy extendido, es decir, que la innovación influye fuertemente en la varianza explicada en las tasas de rentabilidad de las empresas, el apoyo a la idea de que la política del gobierno que promueve la innovación puede tener un impacto significativo en el rendimiento de la empresa. 


\section{Eco-innovación}

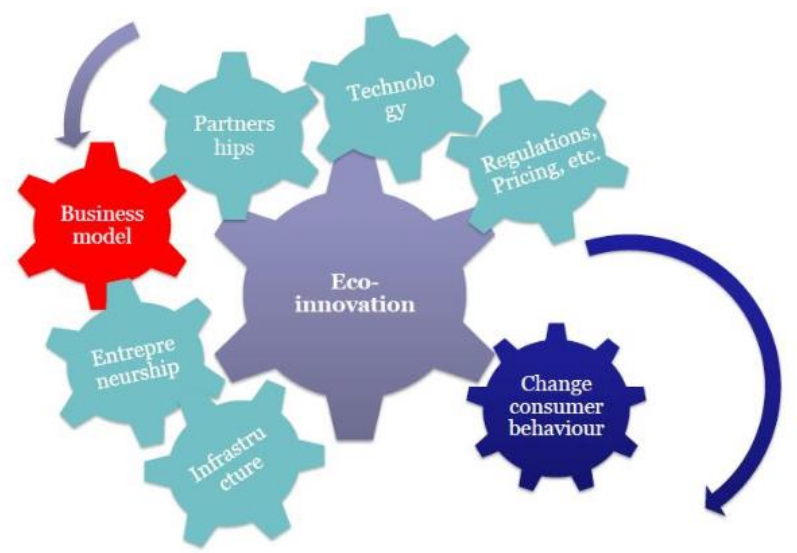

Figura 3 Eco-Innovation Model, Kunapatarawong, 2014

La eco-innovación tiene como principal objetivo el crear valor tanto económico como medioambiental, donde los negocios modelos actúan como palanca de valor y habilitador de tecnologías y soluciones verdes. El enfoque en los negocios modelos permiten una mejor comprensión de cómo se captura el valor ambiental, se convirtió en productos y servicios rentables, y ofrece la comodidad y la satisfacción de los usuarios.

En términos concretos, el análisis de los casos de innovación ecológica puede arrojar luz sobre si, en qué medida y qué valores ambientales se reflejan en firmes proposiciones de valor, la segmentación de clientes, el uso de los recursos, patrones de colaboración y la gestión de los flujos de costos e ingresos, mediante la sustitución de las prácticas comerciales antiguas. Innovadores modelos de negocio también permiten a las empresas a reestructurar su cadena de valor y generar nuevos tipos de relaciones entre productores y consumidores, y alterar la cultura del consumo y las prácticas de uso. Son varios los factores alrededor de un modelo de eco-innovación.

La propuesta conceptual marco de cualquier modelo de eco-innovación debería arrojar luz sobre cómo la lógica de diseño del modelo de negocio puede incorporar la sostenibilidad y orientarse hacia las ecoinnovaciones radicales. A fin de que hacerlo, el marco conceptual va más allá de analizar los modelos de negocio de empresas individuales e incorpora la perspectiva de la cadena de valor.
El enfoque también incluye la noción de impactos más amplios y condiciones macro, tales como los reglamentos pertinentes y el acceso a la financiación, a fin de comprender mejor lo que impulsa a las empresas, considerando la radical innovación ecológica como una propuesta de valor alternativa viable.

\section{Monitoreo y evaluación del impacto de la innovación}

\begin{tabular}{|c|c|c|}
\hline 'Structure' & 'Conduct' & 'Performance' \\
\hline $\begin{array}{l}\text { IP 'structure' } \\
\text { - Membership } \\
\text { composition and } \\
\text { diversity } \\
\text { - Decision making } \\
\text { process } \\
\text { - Committees } \\
\text { - Source of funding } \\
\text { Staff availability } \\
\text { Individual 'structure' } \\
\text { - Type of chain } \\
\text { stakeholder } \\
\text { - Gender } \\
\text { - Level of education } \\
\text { Indicator of wealth } \\
\text { - External environment } \\
\text { - Legal and regulatory } \\
\text { framework }\end{array}$ & $\begin{array}{c}\text { 'Conduct' of IP members } \\
\text { Information sharing } \\
\text { Communication } \\
\text { Coordination } \\
\text { Joint planning } \\
\text { Trust }\end{array}$ & $\begin{array}{l}\text { Value chain 'performance' } \\
\text { - Advocacy } \\
\text { - Collective promotion } \\
\text { - Reint quality standards } \\
\text { Research \& } \\
\text { - Cevelopment } \\
\text { - Markity building } \\
\text { - Arbitration of chain } \\
\text { conflict } \\
\text { - Limiting transaction } \\
\text { costs } \\
\text { - Setting concerted } \\
\text { marketing objectives } \\
\text { Other objectives set by IP }\end{array}$ \\
\hline
\end{tabular}

Figura 4 A conceptual framework to evaluate the impact of innovation platforms on agrifood value chains development (Moreno, Otavo y Castro, 2015)

Elementos de un marco conceptual para monitorear y evaluar el impacto de la innovación plataformas para el desarrollo de cadenas de valor. La estructura de la plataforma se puede caracterizar por su organización interna. El entorno externo a la plataforma también puede ser parte de la estructura dentro de este modelo.

Algunas de las características de los distintos miembros de la plataforma también pueden ser asignadas como un elemento de la estructura para el modelo, es decir, el tipo de los principales interesados dentro de la cadena de valor y algún indicador de la posición de la parte demandada en la sociedad: de género, edad, origen étnico, y un proxy local relevante para la riqueza (Cadilhon, 2013).

La integración de la cadena de suministro se considera de gran importancia estratégica, por eso existen diversas razones y beneficios para que los actores involucrados deseen integrarse interna o externamente, aun cuando se han reportado algunos problemas de la integración como: 
Diferencias en los intereses de los miembros, conflictos entre los objetivos de cada agente, desacuerdos sobre la toma de decisiones y acciones conjuntas, falta de transparencia de la información y altos costos de inversión para implementar sistemas de información entre las organizaciones (Moreno, Otavo y Castro, 2015). El modelo describe la relación que guarda la estructura con la conducta y el desempeño, En las cadenas agroindustriales la principal barrera es la complejidad de la industria y la heterogeneidad de su estructura, debido a que está compuesta por una gran cantidad de agentes, lo que resulta en numerosas interacciones, volviendo problemático el intercambio de información y aumentando la posibilidad de incompatibilidad, desde el punto de vista tecnológico y de los sistemas para compartir información.

Asimismo, en las cadenas agrícolas existe asimetría en el poder ya que las empresas con mayor dominio lo ejercen imponiendo las reglas de colaboración, lo cual aumenta continuamente el desbalance de los beneficios impidiendo que los demás agentes busquen integrarse (Moreno, Otavo y Castro, 2015).

De lo anterior, el modelo de contribución que mejor impacta a la innovación en el sector agroindustrial, depende en gran medida de la estrategia definida para la plataforma que mejor se adapte a la cadena de valor, siendo un modelo contextual a la definición original del comportamiento del productor versus el comportamiento del producto.

Un sistema de medición de desempeño más completo y específico para cadenas agroindustriales fue planteado en 2006 y se sustentó en la revisión de los principales KPI (Key Performance Indicator, por sus siglas en inglés) y los modelos SCOR (Supply Chain Operations Reference, por sus siglas en inglés), Balanced Scorecard, Multi Criteria Analysis, DEA (Data Envelopment Analysis, por sus siglas en inglés), Life Cycle Analysis y Activity Based Costing.

\section{Metodología}

La metodología utilizada para la investigación fue de tipo cuantitativa, transversalcorrelacional.
Fue transversal porque se recolectaron datos del fenómeno, para analizar la interacción de las variables en un momento específico, fue correlacional porque se describió la correlación que guardan las variables de estudio (Hernández Sampieri, Fernández Collado, \& Baptista Lucio, 2010). Las variables que se analizaron en la investigación, se estudiaron en el contexto de las empresas agroindustriales, la investigación se centró en descubrir si existe relación entre estas variables y cómo es esta relación, es decir qué sentido tiene.

La investigación se realizó en cuatro fases, en la fase uno se desarrolló la investigación bibliográfica, en la fase dos se validó y aplicó el instrumento de recolección de datos en empresas del sector agroindustrial, en la fase tres se realizó el análisis de correlación, finalmente se diseñó el modelo. Las variables que se analizaron en la investigación son; cooperación empresarial, capacidad tecnológica, creacion del conocimiento, programas gubernamentales, agrupamientos agroindustriales y diversificación productiva, la investigación se centró en descubrir si existe relación entre estas variables y cómo es esta relación, es decir qué sentido tiene.

Se procedió a realizar una revisión de la literatura científica sobre el tema de innovación agroindustrial en plataformas científicas como Thomson Reuters, Dialnet, Redalyc y Ebso, se considera la literatura de 8 años de antigüedad y se valoró el nivel de impacto de las publicaciones, conforme a dicha revisión se detectaron las 6 variables indicadas anteriormente, se hace hincapié en el hecho de que dichas variables son significativas para la gestión de empresas agroindustriales innovadoras.

Para llevar a cabo el estudio estadístico se elaboró un cuestionario en el que se midieron las variables, la estructura del cuestionario se detalla en los anexos. Es importante comentar que para el estudio estadístico se entrevistaron a productores de noche buena de Atlixco para ello se elaboró un perfil del encuestado que permitió identificar a las personas que participarían en el estudio, las características que deberían cumplir consistía en que fuesen dueños e ingenieros que los apoyaran en la actividad productiva. Se eligieron a estas personas porque son las que tienen mayor conocimiento sobre el tema, en total se entrevistaron a 40 personas. 
Para llevar a cabo las encuestas se solicitó acceso con los productores y se aplicaron personalmente a cada individuo, indicándoles que sus respuestas eran anónimas, para minimizar en lo posible el sesgo. Posteriormente se analizaron estadísticamente los resultados de las encuestas de la etapa anterior, mediante estadística descriptiva y estudios de correlación, para llevar a cabo el análisis se utilizó el software SPSS ${ }^{\circledR}$ versión 19. El estudio de correlación permitió identificar las variables que tienen más nivel de correlación.

En base a los estudios de correlación se determinará cuales variables son más significativas y cuales no, las que sean menos significantes serán excluidas del modelo, mientras que las que presenten mayor correlación serán consideradas para la conceptualización del modelo. La unidad de análisis de la investigación son los productores de noche buena del municipio de Atlixco, que de acuerdo al anuario estadístico de INEGI, se compone de 40 productores.

Correlación de la variable cooperación empresarial en relación al resto de las variables

la variable cooperación empresarial nuestra un coeficiente de correlación con programas gubernamentales de 0.775 , mientras que en relación a creación del conocimiento presenta un valor de 0.754, agrupamientos agroindustriales un valor de 0.791 , capacidad tecnológica 0.789 y diversificación productiva un valor de 0.313 , conforme a los datos abalizados la variable cooperación empresarial nuestra un nivel más alto de correlación con la variable agrupamientos agroindustriales con un valor de 0.791 .

\section{Correlación de la variable programas gubernamentales en relación al resto de las variables}

Respecto a la variable programas gubernamentales los datos indican una correlación con la variable cooperación empresarial de 0.775 , creación del conocimiento 0.657, agrupamientos agroindustriales 0.792, capacidad tecnológica 0.711 y diversificación productiva un valor de 0.327 , dentro de los cuales el mayor valor de correlación es de 0.792 perteneciente a la variable de agrupamientos agroindustriales.

\section{Correlación de la variable creación del conocimiento en relación al resto de las variables}

La variable creación de conocimiento presenta un valor de correlación de 0.754 con la variable cooperación empresarial, programas gubernamentales $0.657, \quad$ agrupamientos agroindustriales 0.756 , capacidad tecnológica 0.805 y finalmente diversificación productiva un valor de 0.319 , el mayor valor de entre las variables corresponde a capacidad tecnológica.

\section{Correlación de la variable agrupamientos agroindustriales en relación al resto de las variables}

Esta variable presenta un valor de correlación con respecto a cooperación empresarial de 0.791, programas gubernamentales 0.792, creación de conocimiento 0.756 , capacidad tecnológica 0.745 y diversificación productiva un valor de 0.335 , el valor máximo de correlación es representado por la variable programas gubernamentales.

\section{Correlación de la variable capacidad tecnológica en relación al resto de las variables}

La presente variable presenta un valor de correlación con la variable 0.789 con la variable cooperación empresarial, programas gubernamentales 0.711 , creación de conocimiento $\quad 0.805, \quad$ agrupamientos agroindustriales 0.745 y diversificación con un valor de 0.315 , el valor máximo de correlación pertenece a la variable creación de conocimiento

\section{Correlación de la variable diversificación productiva en relación al resto de las variables}

Esta última variable presenta valores de correlación bajos en relación al resto de las variables; cooperación empresarial 0.313, programas gubernamentales 0.327 , creación de conocimiento $0.319, \quad$ agrupamientos agroindustriales 0.335 , y finalmente capacidad tecnológica 0.315 , en mayor valor es el perteneciente a la variable programas gubernamentales, sin embargo estos niveles de correlación son significativamente bajos en relación al resto de las variables, lo cual sugiere que esta variable no formaría parte del modelo teórico propuesto. 


\section{Resultados}

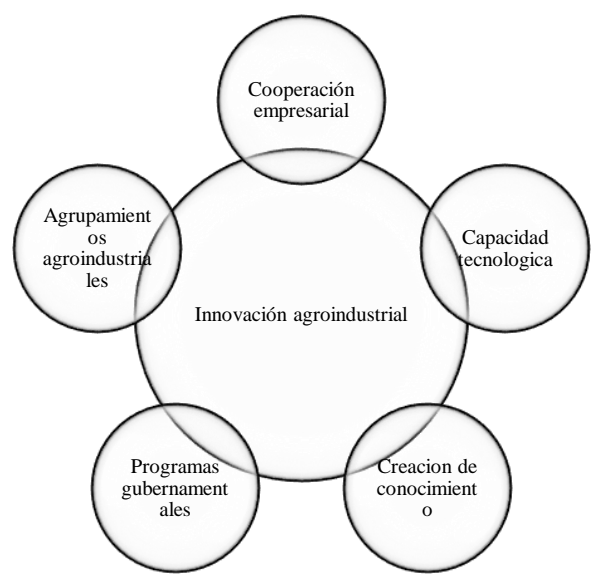

Figura 5 Modelo teórico propuesto para la innovación agroindustrial

Fuente: Elaboración Propia, 2019

La construcción del modelo se fundamenta en los resultados de la investigación estadística y revisión de la literatura científica referente al tema de innovación agroindustrial.

La variable cooperación empresarial se correlaciona positivamente y significativamente en un rango de 0.754 a 0.791 con las variables; programas gubernamentales, creación del conocimiento, agrupamientos agroindustriales, capacidad tecnológica, se puede determinar conforme a la evidencia estadística que arroja la investigación que dichas variables pueden trabajar de forma integral posibilitando la conceptualización teórica de la presente propuesta de modelo.

Respecto a la variable diversificación productiva los datos de correlación son considerados bajos $y$ por ende poco significativos presentando valores de corrección de 0.313 a 0.335 , por lo cual la presente evidencia estadística faculta el hecho que la dicha variable puede ser excluida del modelo, esta situación se presenta con respecto a todas las variables.

La variable programas gubernamentales se correlaciona positiva y significativamente en un rango de 0.657 a 0.792 con las variables: cooperación empresarial, creación del conocimiento, agrupamientos agroindustriales y capacidad tecnológica, se puede determinar conforme a la evidencia estadística que arroja la investigación que dichas variables pueden trabajar de forma integral posibilitando la conceptualización teórica de la presente propuesta de modelo.
La variable creación del conocimiento se correlaciona positiva y significativamente en un rango de 0.657 a 0.805 con las variables: cooperación empresarial, programas gubernamentales, agrupamientos agroindustriales y capacidad tecnológica; se puede determinar conforme a la evidencia estadística que arroja la investigación que dichas variables pueden trabajar de forma integral posibilitando la conceptualización teórica de la presente propuesta de modelo.

La variable agrupamientos agroindustriales se correlaciona positiva y significativamente en un rango de 0.745 a 0.792 con las variables: cooperación empresarial, programas gubernamentales, creación de conocimiento y capacidad tecnológica; se puede determinar conforme a la evidencia estadística que arroja la investigación que dichas variables pueden trabajar de forma integral posibilitando la conceptualización teórica de la presente propuesta de modelo.

La variable capacidad tecnológica se correlaciona positivamente y significativamente en un rango de 0.711 a 0.805 con las variables; cooperación empresarial, programas gubernamentales, agrupamientos agroindustriales, agrupamientos agroindustriales, se puede determinar conforme a la evidencia estadística que arroja la investigación que dichas variables pueden trabajar de forma integral posibilitando la conceptualización teórica de la presente propuesta de modelo.

La variable diversificación productiva como se argumentó anteriormente presente un nivel de correlación bajo con respecto al resto de las variables por lo tanto no se considera dentro de la conceptualización del modelo.

\section{Agradecimientos}

Un agradecimiento al Instituto Tecnológico Superior de San Martín Texmelucan por las facilidades brindadas para el presente trabajo de investigación a si mismo al cuerpo académico de Optimizacion de procesos de manufactura de la carrera de ingeniería industrial. 


\section{Conclusiones}

La presente propuesta surgió derivado de la realización del estado del arte en el cuyo objetivo principal es detectar lo que los científicos denominan " un hueco en la ciencia, se procedió a realizar dicho estado del arte en las plataformas científicas Thompson Reuters, Scopus, Dialnet Redalyc y Ebsco, evidentemente éstas plataformas albergan una infinidad de artículos científicos, se tomó en consideración el nivel de impacto de las revistas en donde fueron publicados, aunado a esta situación, también se tomó en consideración el año de las publicaciones.

Se recomienda en estos términos considerar las publicaciones en un rango máximo de antigüedad de 5 años, sin embargo, en este sentido deben considerarse algunas publicaciones denominadas como "clásicos", en este caso, la literatura de Schumpeter y documentos importantes como el manual de Oslo. Derivado de la revisión de la literatura se detectaron seis variables potenciales: cooperación empresarial, programas gubernamentales, capacidad tecnológica, creación de conocimiento, agrupamientos agroindustriales y diversificación productiva, hasta el momento la literatura científica considera que dichas variables son importantes dentro del ámbito de la innovación agroindustrial, sin embargo hasta el momento habían sido estudiadas de forma aislada, individual, por lo cual el aporte a la ciencia por parte de la presente propuesta en analizarlas de forma integral con el objetivo de determinar si dichas variables pudieran trabajar en conjunto propiciando la posibilidad de conceptualizar un modelo integral teórico de innovación agroindustrial.

Como se describe anteriormente dicho modelo va enfocado al sector agroindustrial, dicho sector tienen un considerable número de actividades económicas tales como; producción de productos cárnicos, alimentos vegetales, alimentos procesados, entre otros, también la producción de flores de ornamento está considerada dentro de este sector, adicionalmente se tiene relación con las empresas productoras de flor de noche buena lo cual posibilita la obtención de datos necesarios, en este caso la población sujeta de estudio son los productores de flor de noche buena denominada Euphorbia pulcherrima del municipio de Atlixco.
Se procedió a buscar en fuentes primarias el número de productores existentes en la zona por lo cual el resultado encontrado fue de 40 productores, en base a esta situación se procedió a diseñar el instrumento de medición, en este caso un cuestionario con preguntas estructuradas y con una escala liker de 5 elementos ( siempre, la mayoría de la veces, a veces, pocas veces, nunca), se procedió a la validez del instrumento mediante el cálculo de alfa de Crombach mediante el software estadístico SPSS el resultado fue de 0.918, posteriormente se aplicaron dichas encuestas obteniendo los datos necesarios para las pruebas de hipótesis en total se formularon 30 hipótesis cada variable con 5 hipótesis respectivamente, al realizar el análisis estadístico se detectó que los datos no presentaban un comportamiento de distribución normal tomando como indicador para esto la desviación típica lo cual fue considerado para determinar que el modelo estadístico ideal para el análisis correlación fuese el Spearman.

Los datos obtenidos de dicho análisis correlacional muestran que las variables; cooperación empresarial, programas gubernamentales, creación del conocimiento, capacidad tecnológica y agrupamientos agroindustriales muestran una correlación de 0.625 a 0.805 lo cual posibilitó la conceptualización del presente modelo propuesto, sin embargo la variable diversificación productiva muestra bajos niveles de correlación de 0.313 a 0.327 lo cual sugiere excluir dicha variable del modelo.

\section{Trabajo Futuro}

La presente propuesta solo se enfocó al analisis correlacional entre si de las varibales sujetas de estudio, bajo esa primicia se realizó la propuesta del presente modelo teoricó, sin embargo, se recomienda como trabajo futuro complementar dicha investigación con un trabajo que evalúe la efectividad del modelo ya aplicado en las empresas agroindustriales, ya que el objetivo principal de dicho modelo es incrementar su nivel competitivo. 


\section{Referencias}

Andrés Felipe Ruiz Moreno, Andrés Leonardo, Caicedo Otavo Javier Arturo Orjuela Castro (2015) External Integration on Agri-Food Supply Chain: A review to the state of the art. Ingeniería Vol. 20 No. 2 Universidad Distrital FJC.

Bryman, A. (2008) Métodos de investigación social (Oxford, University Press).Hernandez Sampieri, R. (2009) Metodología de la Investigación (México, Mc Graw Hill).

Cadilhon, J. J. (2013). A conceptual framework to evaluate the impact of innovation platforms on agrifood value chains development.

Carla Susana Marques, Chris Gerry, Susana Covelo (2011) Innovation and the performance of Portuguese businesses: a 'Sure' approach Int. J. Management and Enterprise Development, Vol. 10, Nos Christian Horn, Alexander Brem, (2013). "Strategic directions on innovation management - a conceptual framework", Management Research Review, Vol. 36 Iss: 10, pp. $939-954$.

Cummings, A. (2013). Construyendo capacidades de innovación en iniciativas asociativas de pequeñas agroindustrias rurales en El Salvador. Revista Iberoamericana de Ciencia, Tecnología y Sociedad - CTS, Septiembre-, 295-319.

Kunapatarawong, R. (2014). Three essays on environmental innovation and corporate social responsibility.

Pérez Pérez, Mario F, Medina García, Victor H (2013) Knowledge Management Model for Fruit-Horticultural Agroindustry Case: Córdoba - Colombia International Journal of Future Computer and Communication.

Zarazúa, J. A., Solleiro, J. L., Altamirano, C. R., Castañón, I. R., y Rendón M. R., (2009).Esquemas de innovación tecnológica y su transferencia en las agroempresas frutícolas del estado de Michoacán. Estudios sociales (Hermosillo, Son.), 17(34), 37-71. 